References:

[1] Mackie SL et al. Can the prognosis of polymyalgia rheumatica be predicted at disease onset? Results from a 5-year prospective study. Rheumatology (Oxford). 2010;49(4):716-22.

[2] Dejaco $\mathrm{C}$ et al. Current evidence for therapeutic interventions and prognostic factors in polymyalgia rheumatica: A systematic literature review informing the 2015 EULAR/ACR. ARD. 2015;74: 1808-17.

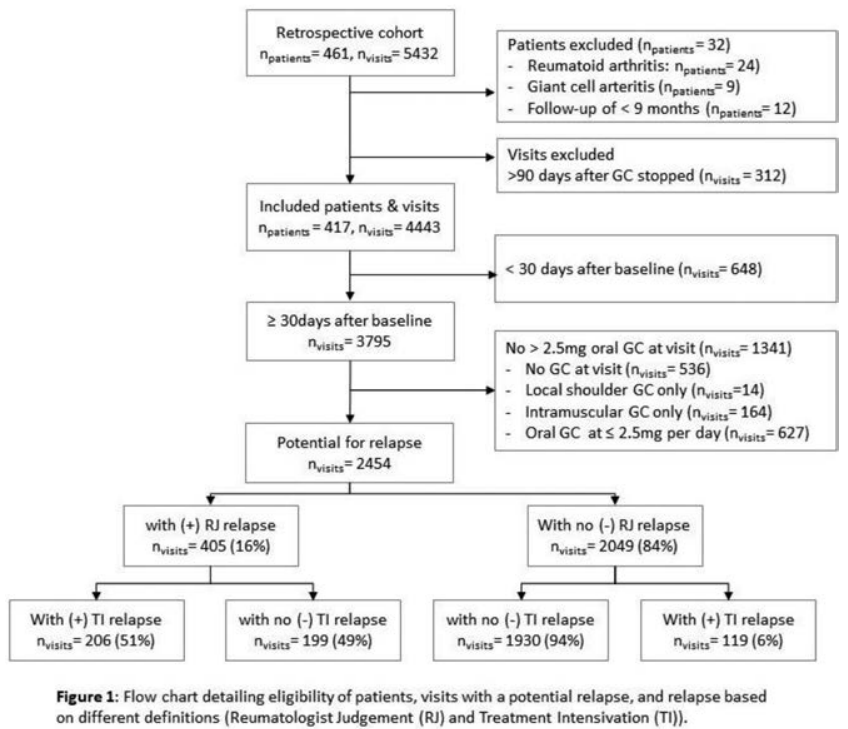

Disclosure of Interests: None declared

DOI: 10.1136/annrheumdis-2020-eular.3560

\section{OP0073 $\quad$ RISK OF DEVELOPING RHEUMATIC DISEASES IN PATIENTS WITH PALINDROMIC RHEUMATISM IN SOUTH KOREA: A NATION-WIDE POPULATION- BASED STUDY}

J. K. Ahn ${ }^{1}$, J. Hwang ${ }^{2}$, J. Lee ${ }^{3}$, H. Kim ${ }^{3}$, G. H. Seo ${ }^{4} .{ }^{1}$ Kangbuk Samsung Hospital, Sungkyunkwan University School of Medicine, Division of Rheumatology, Department of Internal Medicine, Seoul, Korea, Rep. of (South Korea); ${ }^{2}$ Samsung Changwon Hospital, Sungkyunkwan University School of Medicine, Division of Rheumatology, Department of Internal Medicine, Changwon-si, Korea, Rep. of (South Korea); ${ }^{3}$ Samsung Medical Center, Sungkyunkwan School of Medicine, Department of Medicine, Seoul, Korea, Rep. of (South Korea); ${ }^{4}$ Health Insurance Review and Assessment Service, Wonju, Korea, Rep. of (South Korea)

Background: Palindromic rheumatism (PR) has known to be three patterns of disease course: clinical remission of attacks, persistent attacks, and evolution to chronic arthritis or systemic disease. The spectrum in progression to chronic diseases of PR, however, is quite variable; rheumatoid arthritis (RA), systemic lupus erythematosus (SLE), systemic sclerosis (SSc), Sjögren's syndrome (SjS), ankylosing spondylitis (AS), relapsing polychondritis (RP), Behçet's disease (BD), sarcoidosis, and psoriatic spondylitis and arthropathy. Because of the small numbers in case-control studies and too aged investigations, now we needs to shed new light on the fate of PR.

Objectives: The aim was to investigate the epidemiology of PR and the risk of developing various rheumatic diseases compared with non-PR individuals, employing the National Health Insurance Service (NHIS) medical claims data, which covers all medical institutions of South Korea.

Methods: The study used 2007-2018 claims data from the Korean Health Insurance Review and Assessment Service (HIRA). The identified 19,724 PR patients from 2010 to 2016 were assessed for the incidence rate (IR) compared with the population in the given year by 100,000 person-year (py). The date of diagnosis was the index date. After matching with non-PR individuals (1:10) for age, sex and the year of index date, we calculated the hazard ratios (HRs) with 95\% confidence intervals (Cls). The risk of developing the various rheumatic diseases and adult immunodeficiency syndrome (AIDS) as the outcome diseases in PR cohort was estimated. This risk was compared with that of matched non-PR cohort.

Results: Of 19,724 PR patients (8,665 males and 11,059 females), the mean age was $50.2 \pm 14.9$ years $(47.7 \pm 14.4$ years in males and $52.6 \pm 14.9$ years in females, $p<0.001)$. The ratio of male to female patients with $\mathrm{PR}$ was approximately $1: 1.28$. The annual IR of PR was $7.02(6.92-7.12)$ per 100,000 py (6.22 (6.09-6.35) and $7.80(7.66-7.95)$ per 100,000 py in males and females, respectively). The mean duration to develop the outcome diseases was significantly shorter in PR cohort compared that of non-PR cohort (19.4 vs. 35.8 months, $p<0.001)$. The most common outcome disease was RA $(7.34 \%$ of PR patients; $80.0 \%$ of total outcome diseases), followed by AS, SLE, BD, SjS MCTD, DM/PM, SSc, RP, psoriatic arthropathy, and AIDS in PR cohort. The patients with PR had an increased risk of RA (HR 46.6, 95\% Cl [41.1-52.7]) psoriatic arthropathy (44.79 [15.2-132.4]), SLE (24.5 [16.2-37.2]), MCTD (22.0 [7.7-63.3]), BD (21.0 [13.8-32.1]), SjS (12.4 [8.5-17.9]), AS (9.0 [6.7-12.2]), DM/ PM (6.1 [2.6-14.8]), and SSc (3.8 [1.5-9.6]) but not of AIDS. The risk of developing RA was greater in male patients (HR 58.9, 95\% Cl [45.6-76.2] vs. 43.2 [37.4-49.8], $p$ for interaction $=0.037$ ) while female patients encountered a higher risk of developing AS (15.8 [8.9-28.1] vs. 7.2 [5.0-10.3], $p$ for interaction $=0.023$ ). The risk of developing RA, SLE, SjS, and BD were significantly more highly affected in younger age $(p$ for interaction $<0.001,=0.003,0.002$, and 0.017 , at each).

Conclusion: This nationwide, population-based cohort study demonstrated that patients with PR had an increased risk of developing various rheumatic diseases, not only RA but also psoriatic arthropathy. Therefore, patients with PR needs to be cautiously followed up for their potential of diverse outcome other than RA: RA, SLE, SjS, and BD in younger patients, RA in males, and AS in females, in particular.

Disclosure of Interests: None declared

DOI: 10.1136/annrheumdis-2020-eular.3664

\section{OP0074 MULTIMORBIDITY CLUSTERS, DETERMINANTS AND TRAJECTORIES IN OSTEOARTHRITIS IN THE UK: FINDINGS FROM THE CLINICAL PRACTICE RESEARCH DATALINK}

S. Swain ${ }^{1}$, C. Coupland ${ }^{2}$, V. Strauss ${ }^{3}$, C. Mallen ${ }^{4}$, C. F. Kuo ${ }^{5}$, A. Sarmanova ${ }^{6}$, M. Doherty ${ }^{1}$, W. Zhang ${ }^{1}{ }^{1}$ University of Nottingham, Academic Rheumatology, Nottingham, United Kingdom; ${ }^{2}$ University of Nottingham, Division of Primary Care, Nottingham, United Kingdom; ${ }^{3}$ University of Oxford, NDORMS, Oxford, United Kingdom; ${ }^{4}$ Keele University, School of Primary, Community and Social Care, Keele, United Kingdom; ${ }^{5}$ Chang Gung Memorial Hospital, Division of Rheumatology, Allergy and Immunology, Taiwan, Taiwan, Republic of China; ${ }^{6}$ University of Bristol, Musculoskeletal Research Unit, Bristol Medical School, Bristol, United Kingdom

Background: Multimorbidity ( $\geq 2$ chronic conditions) escalates the risk of adverse health outcomes. However, its burden in people with osteoarthritis (OA) remains largely unknown.

Objectives: To identify the clusters of patients with multimorbidity and associated factors in OA and non-OA populations and to estimate the risk of developing multimorbidity clusters after the index date (after diagnosis).

Methods: The study used the Clinical Practice Research Datalink - a primary care database from the UK. Firstly, age, sex and practice matched OA and non-OA people aged 20+ were identified to explore patterns and associations of clusters of multimorbidity within each group. Non-OA controls were assigned with same index date as that of matched OA cases. Secondly, multimorbidity trajectories for 20 years after the index date were examined in people without any comorbidities at baseline in both $\mathrm{OA}$ and non-OA groups. Latent class analysis was used to identify clusters and latent class growth modelling was used for cluster trajectories. The associations between clusters and age, sex, body mass index (BMI), alcohol use, smoking habits at baseline were quantified through multinomial logistic regression.

Results: In total, 47 long-term conditions were studied in 443,822 people (OA221922; non-OA- 221900), with a mean age of 62 years (standard deviation \pm 13 years), and $58 \%$ being women. The prevalence of multimorbidity was $76.6 \%$ and $68.9 \%$ in the OA and non-OA groups, respectively. In the OA group five clusters were identified including relatively healthy (18\%), "cardiovascular (CVD) and musculoskeletal (MSK)' (12.3\%), metabolic syndrome (28.2\%), 'pain and psychological (9.1\%), and 'musculoskeletal' (32.4\%). The non-OA group had similar patterns except that the 'pain+ psychological' cluster was replaced by 'thyroid and psychological'. (Figure 1) Among people with OA, 'CVD+MSK' and metabolic syndrome clusters were strongly associated with obesity with a relative risk ratio (RRR) of $2.04(95 \% \mathrm{Cl} 1.95-2.13)$ and $2.10(95 \% \mathrm{Cl} 2.03-2.17)$, respectively Women had four times higher risk of being in the 'pain+ psychological' cluster than men when compared to the gender ratio in the healthy cluster, (RRR 4.28; $95 \% \mathrm{Cl} 4.09-4.48)$. In the non-OA group, obesity was significantly associated with all the clusters. 